\title{
DIE VERMESSUNG ÄSTHETISCHER ERSCHEINUNGEN
}

Ob und wie man ästhetische Objekte vermessen darf oder soll, ist Gegenstand von Grundsatzdebatten. Wer die eigentümliche Unschärfe, die allen künstlerischen Werken eignet, in messbare Einheiten zerlegen will, setzt sich leicht dem Verdacht des reduktionistischen Positivismus aus. Ungeachtet einer langen, bis an die Wende zum 20. Jahrhundert zurückreichenden Reihe von Versuchen, die philosophische Ästhetik auf eine empirisch-naturwissenschaftliche Basis zu stellen, zu denen nicht zuletzt auch die experimentalpsychologischen Studien aus dem Umfeld der Filmologie-Bewegung der I95oer Jahre zählen, scheint sich zwischen Empirie und Ästhetik weiterhin ein kaum zu überwindendes Spannungsfeld aufzutun.

Dennoch stellen sich in der Filmwissenschaft sowie in jenen Zweigen der Medienwissenschaft, die sich mit der ästhetischen Produktion in und durch Medien beschäftigen, mitunter Fragen, die den Einsatz empirischer Werkzeuge und Methoden - zu denen heute insbesondere auch computergestützte Verfahren zählen - zumindest nahelegen. Dies gilt insbesondere für die Analyse großer, meist diachroner Korpora, die hinsichtlich bestimmter Gestaltungsformen oder ästhetischer Strategien untersucht werden sollen. Solche breit angelegte, umfassende Studien profitieren von einem computergestützten Analysetool, das idealerweise an eine öffentlich zugängliche, webbasierte Applikation angebunden wäre, sodass jeder einzelne Forscher nicht nur seine Ergebnisse kollaborativ in diese Datenbank eintragen könnte, sondern gleichzeitig verschiedene Parameter zur Kontextualisierung seiner Analyse abrufen und somit das einzelne Werk historisch, institutionell oder mit Blick auf den Personalstil einer Kameraperson oder eines Regisseurs einordnen könnte.

Mit dieser Erweiterung von traditioneller qualitativer Analyse an einzelnen Werken zu groß angelegten, kollaborativen Studien, die sich empirischer Werkzeuge bedienen, sich aber dennoch einer geisteswissenschaftlichen Tradition verpflichtet sehen, stellen sich neue methodische Herausforderungen. Denn wenn der Horizont der Arbeit eines Einzelnen an einem Korpus über- 
schritten wird, werden strenge Anforderungen an eine systematische Erfassung und Beschreibung von medialen Eigenheiten oder Effekten notwendig, welche einerseits eine möglichst genaue Standardisierung der Beobachtungen sicherstellt, andererseits aber die Ergebnisse auch in ein robustes Framework einbettet, in dem sie intersubjektiv kommunizierbar werden. In computergestützten Verfahren ist es notwendig, die «filmische Form in informatikkompatible, $<$ maschinenlesbare> Termini ${ }^{1}$ umzukodieren. Genau dieses Problemfeld zwischen individuellem Zugang zur qualitativen Erfassung ästhetischer Parameter und deren Normierung in einem für computergestützte Verfahren tauglichen Rahmen ist die übergeordnete Thematik dieses Texts, in dessen Zentrum eine Fallstudie zur Entwicklung eines Verfahrens der Farbanalyse einige grundlegende epistemologische und wissenschaftstheoretische Aspekte verdeutlichen wird. Am Ende lassen sich summarisch die filmgeschichtlichen Entwicklungen und medientheoretischen Implikationen in einen Zusammenhang stellen, die verdeutlichen, welche Grenzen einem empirischen Zugriff auf ästhetische Erscheinungen gesetzt sind und welche Möglichkeiten darin gleichzeitig begründet liegen.

\section{Von der experimentellen Ästhetik zu Digital Humanities 2.0}

Ein kurzer Abriss der disziplinären Entstehung von empirischen Ansätzen der ästhetischen Forschung zeigt auf, wie sich aus dem ursprünglich psychologischen Zugang eine geisteswissenschaftlich orientierte Linie werkimmanenter Analyse mit formalistischen oder strukturalistischen Methodiken und schließlich eine neue Tradition der computergestützten kollaborativen Zusammenarbeit mit webbasierten Interfaces entwickelt hat, die Digital Humanities 2.o. ${ }^{2}$ Damit wird deutlich, dass die Empirie im Zeichen einer übergeordneten philosophischen Ästhetik einer geisteswissenschaftlichen Tradition verpflichtet ist, die sich von den sozialwissenschaftlichen Herangehensweisen klar unterscheidet.

Eine erste Hochblüte empirischer Ansätze in der ästhetischen Forschung war in der zweiten Hälfte des I9. Jahrhunderts zu beobachten, mit Gustav Theodor Fechner als dem prominentesten Vertreter. Sein Ziel - formuliert in Zur experimentalen Asthetik ${ }^{3}$ und vor allem in der Vorschule der Ästhetik ${ }^{4}$ - war es, über die Vermessung von ästhetischen Objekten allgemein gültige Regeln für das objektiv Schöne zu finden. Bei aller teilweise berechtigten Kritik an Fechners normativem Ansatz ${ }^{5}$ darf nicht vergessen werden, dass diese Epoche und vor allem auch Fechners eigene Forschung einen unglaublichen Fortschritt hervorgebracht haben. Die Psychophysik mit ihrer systematischen, empirisch gestützten Untersuchung des Verhältnisses von Reiz und Empfindung in der menschlichen Wahrnehmung hat nachhaltig die Entwicklung jener technischen Medien gestützt oder sogar überhaupt ermöglicht, die sich zuerst an die Sinne richten, also namentlich des Films als Leitmedium des 20 . Jahrhunderts.
1 Stefan Hahn, Filmprotokoll Revisited. Ground Truth in Digital Formalism, in: Klemens Gruber, Barbara Wurm (Hg.), Digital Formalism, Die kalkulierten Bilder des Dziga Vertou, Wien (Böhlau) 2009 (Maske und Kothurn, Bd. Bd. 55, Nr. 3), 129-136.

2 Siehe die Konferenz Digital Humanities 2.0, die im Februar 2011 an der Harvard University veranstaltet wurde: http://metalab.haruard. edu/2011/02/09/digital-humanities2-o-emerging-paradigms-in-the-artsand-humanities-in-the-informationagel, gesehen am 24.5.2011, oder verschiedene Projekte an der Stanford University, http://humanexperience.stanford.edu/digital, gesehen am 4.7.2011.

3 Gustav Theodor Fechner, Zur experimentalen Ästhetik, Leipzig 1871 , http:|/www.uni-leipzig.de/ffipsycho/ wundt/opera/fechner/expaesth/E1Aesthl. htm, gesehen am 2.1.2011.

4 Gustav Th. Fechner, Vorschule der Ästhetik, Leipzig (Breitkopf) 1876, http:||www.archive.org/details/ vorschulederaest12 fechuoft, gesehen am 2.1.2011.

5 Christian G. Allesch, «Fechners Ästhetik - eine Provokation?», Vortrag beim Ehrensymposion zum 200. Geburtstag von Gustav Theodor Fechner, Leipzig, 19.-20.10.2001, http://www.uni-salzburg.at/pls/portal/ docs/1/347564.DOC, gesehen am 3.1.2011. 
6 Siehe dazu die Diagramme, die von Dziga Vertov erstellt wurden, und insbesondere deren theoretische Aufarbeitung in Barbara Wurm, Vertov digital. Numerisch-grafische Verfahren der formalen Filmanalyse, in: Gruber, Wurm (Hg.), Digital Formalism, 15-43. Mit der Rolle von Diagrammen zur Visualisierung von Strukturen werde ich mich an anderer Stelle befassen. Ähnliche Partituren finden sich in Eisensteins Werk, so in seinem Aufsatz «Vertikalmontage», in: Sergej M. Eisenstein, Jenseits der Einstellung. Schriften zur Filmtheorie, hg. von Felix Lenz und Helmut H. Diederichs, Frankfurt/M. (Suhrkamp) 2006 [1940/41].

7 Wurm, Vertov digital, 41.

8 Wassily Kandinsky, Punkt und Linie zu Fläche. Beitrag zur Analyse der malerischen Elemente, Bern (Benteli) 1964 [1926].
Eine zweite Blüte von anderer Gestalt fand in den I920er Jahren statt, und zwar vor allem in der russischen Avantgarde, aber auch in anderen europäischen Bewegungen, so im Umfeld des Bauhauses. Nun waren es die Künstler selbst - Wassily Kandinsky, Paul Klee, Johannes Itten, Dziga Vertov, ${ }^{6}$ Sergej Eisenstein - die, teilweise gestützt auf Einsichten aus der physiologischen und psychologischen Forschung von Iwan Pawlow und Lew Wygotski, auf der Suche nach allgemeinen Prinzipien künstlerischer Gestaltung waren.

Die Zähmung analoger Filmkontinuen in den numerischen, graphischen und diagrammatischen Skripten, die - um es mit Nelson Goodman zu formulieren - meist syntaktisch und semantisch disjunkte Elemente generieren, stellt den <freien Kreativgeist> der russischen Avantgarde radikal in Frage, ${ }^{7}$

schreibt Barbara Wurm zu diesem notorischen Spannungsfeld zwischen Kunst und Vermessung.

Auslöser für die Konjunktur solcher Verfahren waren unter anderem die Anforderungen der abstrakten Malerei und des experimentellen Films, die mit ihrer Nichtreferenzialität ihre Wirkung ausschließlich oder hauptsächlich aus kompositorischen Strategien entwickeln konnten. Vorbild einer dermaßen strukturierten Gestaltungsweise war die Musik mit ihren während Jahrhunderten entwickelten Gesetzen der Harmonielehre und einer streng geregelten, expliziten Notation. Sicherlich einer der unangefochtenen Vertreter einer analytischen Theoriebildung in der Kunst war Kandinsky, der 1926 in Punkt und Linie zu Fläche schrieb:

Die bis heute herrschende Behauptung, es wäre verhängnisvoll, die Kunst zu <zerlegen〉, da dieses Zerlegen unvermeidlich zum Tod der Kunst führen müsste, stammt aus der unwissenden Unterschätzung der bloßgelegten Elemente und ihrer primären Kräfte. ${ }^{8}$

Kandinsky zeigte in eben dieser Schrift auf, wie sich über die Freilegung von elementaren Bausteinen in Praxis und Theorie des Kunstschaffens eine radikal neue Perspektive eröffnete.

An die durch den von Burrhus Frederic Skinner popularisierten und radikalisierten Behaviorismus beförderte Verschiebung in der psychologischen Forschung schloss sich eine neuerliche Welle von experimenteller Forschung an, in deren Zentrum erneut zunehmend die empirische Untersuchung des Verhältnisses von Reiz und Reaktion stand. Anders als in den I92oer Jahren sind es nun nicht die Kunstschaffenden selbst, die sich dieser Werkzeuge bedienen, sondern die Theoretiker. Nur in abgeschwächter Form finden sich diese Einflüsse in geisteswissenschaftlich ausgerichteten theoretischen Strömungen wie dem Strukturalismus oder der Semiotik der i $960 e r$ und I $970 e r$ Jahre, die - ähnlich wie der russische Formalismus in den I920er Jahren - nach grundlegenden Prinzipien der poetischen oder der bildlichen Kommunikation fragten, um daraus ein Fundament zu errichten, um die damals dominierende Hermeneutik zugunsten einer werkimmanenten Analyse in den Hintergrund zu drängen, wie 
es Roland Barthes in seinem einflussreichen Text «Der Tod des Autors» ${ }^{\mathbf{9}}$ verlangte. Nun war es nicht mehr die Musik, sondern die Sprache, ihre syntaktischen und semantischen Strukturen, die als Modell der Analyse galten.

Als David Bordwell und Kristin Thompson ab Ende der I97oer Jahre im sogenannten Wisconson-Projekt ${ }^{\mathbf{1 0}}$ mit der Triade Neoformalismus, Kognitivismus, historische Poetik ein analytisches Instrumentarium postulierten, das teilweise explizit, teilweise implizit auf die hier erwähnten Vorläufer Bezug nahm, wandten sie sich vor allem gegen die damals vorherrschende, ideologiekritisch, marxistisch und feministisch orientierte Ausrichtung in der Filmwissenschaft, die Bordwell $1989^{11}$ sehr dezidiert ablehnte. Im Wesentlichen ging es ihm um die Übermacht dieser theoretischen Ansätze, die er als doktrinäre und spekulative «Grand Theory» kritisierte. Im Unterschied zum Top-down-Approach hermetischer methodischer Systeme, in denen die individuellen Werke gemäß Bordwell nur dazu dienten, die Hypothesen zu stützen und zu illustrieren, machte er sich für einen induktiven Bottom-up-Zugang stark, indem aus der detaillierten Analyse der einzelnen Filme allgemeine Regeln des Zusammenhangs zwischen Gestaltung und Wirkung abzuleiten wären. Als weitere Dimension der Analyse schlug er eine sowohl synchrone als auch diachrone Perspektive vor: Zunächst sollten die Werke in Gruppen einer Epoche zusammengefasst untersucht und anschließend im historischen Vergleich kritische und signifikante Veränderungen dieser Prinzipien herausgearbeitet werden. Wiewohl dieser Ansatz im Kern einer faktografischen Evidenz verpflichtet ist, will Bordwell die Poetik dennoch nicht als rein empirisch verstanden wissen:

A poetics can be rationalist or empiricist, Kantian or phenomenological, deductivist or inductivist, idealist (as Bazin probably was) or positivist (as Barry Salt seems to be) $[\ldots]$ whatever its ontology or epistemology or discovery principles, it requires an appeal to intersubjectively accepted data. ${ }^{12}$

Referenzwerk dieses Vorgehens war Bordwells, Staigers und Thompsons The Classical Hollywood Cinema..$^{13}$ Ziel dieser Publikation war es, die Standards dieser Epoche analytisch zu bestimmen. Im Zentrum der neoformalistischen Analyse des Stils standen devices, also spezifische Gestaltungselemente, die über die basale Arbeit am Korpus hinaus historisch wie institutionell kontextualisiert wurden.

Noch deutlich fokussierter war im Anschluss Barry Salts statistische Filmanalyse eines riesigen Korpus von mehreren roo Filmen aus mehr als 90 Jahren, die er in Film Style and Technology ${ }^{14}$ veröffentlichte, einem Werk, das systematisch das Auftreten bestimmter stilistischer Eigenheiten des Films im Kontext der technologischen Entwicklung aufrollt. Gerade in Salts Publikation wird aber die problematische Seite dieses Ansatzes deutlich, und zwar vor allem dort, wo er sich einzelnen Werken widmet, so in der Abhandlung zu Max Ophüls' Filmen, wo es ihm nicht gelingt, die in der statistischen Auswertung erhobenen Daten zu Schnittfrequenz, Einstellungsgrößen und Kamerabewegungen sinnvoll mit der ästhetischen Wirkung oder der erzählerischen Struktur zu
9 Roland Barthes, Der Tod des Autors [1968], in: Roland Barthes, Das Rauschen der Sprache, Frankfurt/M. (Suhrkamp) 2006, $57-63$.

10 Britta Hartmann, Hans J. Wulff, Vom Spezifischen des Films. Neoformalismus - Kognitivismus - Historische Poetik, in: Montagelav, Nr. 4/1, 1995, 5-22.

11 David Bordwell, Historical Poetics of Cinema, in: R. Barton Palmer (Hg.), The Cinema Text. Methods and Approaches, New York (AMS Press) 1989, 369-398, hier 385 .

12 Bordwell, Historical Poetics of Cinema, 372.

13 David Bordwell, Janet Staiger, Kristin Thompson, The Classical Hollywood Cinema, London (Routledge) 1985.

14 Barry Salt, Film Style and Technology. History and Analysis, London (Starword) 1992. 
15 http:|/www.cinemetrics.lv, gesehen am 25.1.2011.

16 http://www.cinemetrics.lv/cinemetrics.php, gesehen am 25.1.2011.

17 Zum Beispiel für The Conversation (USA 1974, Francis Ford Coppola), erfasst von Pius Meyer, http://www. cinemetrics.lv/movie.php?movie $\mathrm{ID}=4965 \mathrm{H}$.

18 Luchino Viscontis Werk, http:// www.cinemetrics.lv/lab.php?ID=111.

19 Anton Fuxjäger, Wenn Filmwissenschaftler versuchen, sich Maschinen verständlich zu machen. Zur (mangelnden) Operationalisierbarkeit der Begriffs «Einstellung〉 für die Filmanalyse, in: Gruber, Wurm (Hg.), Digital Formalism, 115-127.

20 Siehe exemplarisch Chitra Dorai sowie Svetha Venkatesh (Hg.), Media Computing. Computational Media Aesthetics, Boston (Kluwer Academic Publishers) 2002, sowie Stephen Mamber, Narrative Mapping, in: Anna Everett, John T. Caldwell (Hg.), New Media. Theories and Practices of Digitextuality, New York (Routledge) 2003, 145-158, und weitere seiner Arbeiten auf http:||www.cdh.ucla.edu| people/faculty/stephen-mamber.html, gesehen am 18.6.2011.

21 http:/|www.anvil-software.de, gesehen am 4.7.2011.

22 http://www.iri.centrepompidou. fr/outils/lignes-de-temps/?lang=fr_fr, gesehen am 19.6.2011. verbinden. So wirken diese Analysen seltsam banal und blutleer, sobald sie die Domäne strenger Faktografie verlassen, und es bleiben die wichtigen Fragen offen, nämlich warum, wo und mit welcher Wirkung zum Beispiel die spektakulären Dolly- und Kranfahrten in Max Ophüls' Filmen eingesetzt wurden.

Schnittfrequenzen und Einstellungsgrößen sind inzwischen die Grundpfeiler statistischer Filmanalysen. Auf Yuri Tsivians Website Cinemetrics ${ }^{15}$ steht eine Software ${ }^{16}$ zur Verfügung, mit der man diese Daten erheben und dann in die dort bereitgestellte Datenbank eintragen kann. Daraus entstehen verschiedene Diagramme und Tabellen, so für jeden einzelnen Film wie auch für die Gesamtheit der Filme, in deren Verteilung der durchschnittlichen Einstellungsdauer (average shot length ASL) sich einzelne Filme ${ }^{17}$ oder Werkgruppen ${ }^{18}$ hervorheben lassen.

Ohne Zweifel ist der Schnittrhythmus ein wesentliches Gestaltungselement des Films, bedeutsam gerade im Zusammenhang mit der Aufmerksamkeit des Zuschauers, mit dramaturgischen Spannungsbögen, aber auch hinsichtlich des von André Bazin beschriebenen Realitätseffekts von Plansequenzen. Es ist dennoch anzumerken, dass sich diese Größen zwar relativ leicht und sehr genau sogar als Bruchteile von Sekunden erfassen lassen, denn es sind ja grundsätzlich objektiv messbare Einheiten, auch wenn sich selbst diese simple Einheit - nämlich die filmische Einstellung - weit schwieriger operationalisieren lässt, als man annehmen könnte. ${ }^{19}$

Dennoch ist damit wenig gesagt über die perzeptive Qualität des Rhythmus, die sich vielmehr auch aus anderen Faktoren speist. So ist die sogenannte Transparenz des Schnitts im Continuity-System ein Aspekt, der mitbestimmt, ob die Schnitte überhaupt auffallen oder ob sie nicht so sehr konventionalisiert sind, dass sie sich viel eher im Wahrnehmungskontinuum auflösen. Schon in diesen relativ banalen, allgemein bekannten Einsichten äußert sich die grundlegende Problematik, dass quantitative Daten in der ästhetischen Forschung nicht isoliert zu betrachten sind.

Spätestens seit Beginn der Jahrtausendwende wird zunehmend Software zur computergestützten Analyse von Filmen entwickelt. ${ }^{20}$ Im Rahmen des Forschungsprojekts Digital Formalism, das sich mit den «kalkulierten Bilder[n] des Dziga Vertov» beschäftigte, kam die Software Anvil zur Anwendung, die ab 2000 an der Universität des Saarlandes entwickelt worden war, um gestische Einheiten zu bestimmen. ${ }^{21}$ Hier schließt eine weitere jüngst publizierte Entwicklung des Institut de Recherche et d'Innovation des Centre Pompidou in Paris an, die Software Ligne de temps, ${ }^{22}$ welche ein solches computergestütztes Analyseinstrument einbettet in ein Sichtungstool, das es dem individuellen Forscher erlaubt, eigene Kommentare, Bilder oder Internetlinks direkt mit dem analysierten Objekt zu verbinden.

Das weitaus anspruchvollste Feld für die ästhetisch orientierte Filmwissenschaft ist die Analyse der Farbe, da neben physiologischen und wahrnehmungspsychologischen auch kulturelle, historisch wandelbare Faktoren sowohl 
in der ästhetischen wie in der analytischen Domäne zu berücksichtigen sind. Es ist hier nicht der Ort, die umfangreichen technischen Aspekte des Farbmanagements zu diskutieren, sondern es stehen die praktischen und theoretischen Anforderungen im Vordergrund, die sich um eine systematische Erfassung von Farbwerten - und in diesem Komplex Graustufen- und Kontrastinformationen - grundsätzlich stellen, eben weil sie sich a priori einer einfachen Matrix versperren.

Ziel der weiteren Abhandlung wird es deshalb sein, diesen viel schwierigeren Bezirk der Ästhetik zu diskutieren, in welchen die Phänomene nicht an sich schon zergliedert sind, sondern sich als kontinuierliche Reizspektren präsentieren und in denen eine sinnvolle Fragmentierung erst gefunden werden soll, nämlich Aspekte der Farbe, der Helligkeit und des Kontrasts. Hintergrund der folgenden Überlegungen ist die Möglichkeit, mit computergestützten, semiautomatisierten Verfahren große Korpora in Grundzügen zu erfassen und für die qualitative Forschung, die unbedingt daran anzuschließen hat, zu öffnen. Für die Analyse wäre es überaus nützlich, wenn man für bestimmte Perioden und - auf einer tiefer gelegenen Ebene für einzelne Filmemulsionen oder Verfahren wie beispielsweise Technicolor - Sets von Parametern bestimmen könnte, um sie im Anschluss auf einer kollaborativen Plattform im Internet zu präsentieren, natürlich so weit wie möglich geleitet von kontextueller Forschung zum einzelnen Werk, zu dessen Rezeption und Produktion sowie zu historischen, politischen und institutionellen Zusammenhängen. Jede individuelle Analyse, so meine These, könnte von Datenbanken profitieren, in denen nach allen Regeln der Kunst Informationen über die Standards einer Epoche gespeichert wären.

Ein solches Projekt steht somit in der erwähnten, noch jungen Tradition von Digital Humanities 2.o, in welcher neue Formen der internetbasierten Zusammenarbeit in der Erfassung und Kommentierung von kulturell bedeutsamen Beständen postuliert werden. Sie fußen insbesondere auf den neueren Ansätzen semantischer Netzwerke, welche assoziative Verknüpfungen anhand von intuitiv zugänglichen, natürlichsprachlichen Verbindungen erlauben, ${ }^{23}$ und nutzen das ungeheure Potenzial zur Bündelung weltweit distribuierter Kompetenz durch die Zusammenarbeit und Dissemination von Wissen im Internet. Solche Ansätze sind notwendigerweise kollaborativ und interdisziplinär. Derzeit sehen wir mehr und mehr Forschungsprojekte, vor allem in den USA, die in diesem intermediären Bereich situiert wären, in welchem semi-automatische Analyseprozesse geleitet von fundierten geisteswissenschaftlichen Betrachtungen zur Anwendung kommen.

Es geht im Folgenden darum, methodische und erkenntnistheoretische Fragen mit einem engeren Fokus auf die Farbfrage exemplarisch abzuhandeln und zu reflektieren, und zwar mit folgenden Schwerpunkten: Segmentierung, Standardisierung, Erfassen von Proportionen, die im Folgenden ausgearbeitet werden. Dabei gilt es anzumerken, dass mit Ästhetik zunächst die
23 Ein exemplarisches Projekt ist e-codices, eine virtuelle Bibliothek mittelalterlicher Handschriften in der Schweiz, die es ausgewählten Teilnehmern erlaubt, die Bestände nicht nur für die eigene Forschung zu sichten, sondern auch zu kommentieren, http:/|www.e-codices. unifr.ch. 
24 Weiterführende Informationen finden sich in Norbert Welsch, Claus Chr. Liebmann, Farben. Natur, Technik, Kunst, Heidelberg (Elsevier, Spektrum Akademischer Verlag) 2004, sowie Heinrich Zollinger, Farbe. Eine multidisziplinäre Betrachtung, Zürich (Verlag Helvetica Chimica Acta) 1999, oder in Günther Kebeck, Bild und Betrachter. Auf der Suche nach Eindeutigkeit, Regensburg (Schnell und Steiner) 2006.

25 Tatsächlich ist diese Zuschreibung von Farbtönen zur spektralen Empfindlichkeit der Zapfen eine grobe Vereinfachung, weil sich diese Spektren deutlich überschneiden und das Maximum des «Rot»Sensors eigentlich im gelb-orangen Bereich liegt (siehe Welsch et al., Farben, 241).

26 Regula Anklin-Mühlemann, Rudolf Gschwind, Script zur Vorlesung Farbphotographie, Universität Basel 2001, 7. Das Script sowie weitere informative Materialien zur fotochemischen und digitalen Bildtechnologie befinden sich auf der Website des Imaging and Media Lab, http:/|www. foto.unibas.ch/index.php?content=22, gesehen am 27.2.2011.

27 Zollinger, Farbe, 109. Zollinger bemerkt allerdings, dass neuere psychophysikalische Untersuchen nahelegen, dass Herings Theorie nicht ganz korrekt ist (vgl. Zollinger, Farbe, 113). material-ästhetische Seite gemeint ist, während die rezeptions-ästhetische Dimension, die eigene qualitative und/oder quantitative Ansätze erfordern würde, ausgeblendet wird.

\section{Segmentierung und Standardisierung}

Jede Analyse ist geleitet von einer Segmentierung. Während - wie erwähnt - die Segmentierung im Fall von Schnittfrequenzen schon in der materiellen Grundlage der Analyse vorhanden ist, müssen wir sie erst festlegen, wenn wir ein kontinuierliches Spektrum von Phänomenen untersuchen. Das gilt im Bereich des Akustischen für die Entwicklung der Dynamik und die Frequenzdistribution, das gilt analog dazu im visuellen Bereich für die Farb-, Helligkeits- und Kontrastinformationen. Sinnvollerweise suchen wir eine Referenz, die schon in der natürlichen Wahrnehmung gegeben ist, und zwar aus folgenden Gründen: Erstens ist eine Gliederung, die schon die Grundlagen der Wahrnehmung berücksichtigt, intuitiv besser nachvollziehbar und liefert zweitens Resultate, die in der Rezeption tatsächlich eine Rolle spielen. Drittens ist immer zu berücksichtigen, dass die audio-visuellen Medien selbst a priori hinsichtlich der Eigenschaften des menschlichen Wahrnehmungssystems ausgelegt worden sind, eine Binsenwahrheit, die in der abbildungstheoretischen Diskussion allzu oft vernachlässigt wird, gerade in technikdeterministischen Ansätzen, die davon ausgehen, dass Technologien wahrnehmungsbestimmend sind.

Wenn also die wahrnehmungspsychologischen Grundlagen determinierend sind für eine sinnvolle Zergliederung, ist ein sehr rudimentärer Rekurs auf die eingangs erwähnten psychophysischen Gesetze unentbehrlich. Dieser Rekurs soll nicht mehr leisten, als eine Grundlage für die weitere Argumentation zu liefern. ${ }^{24}$

Farbtöne (bues) sind in ihrer Wahrnehmung deutlich durch die Beschaffenheit des visuellen Systems bestimmt. Einerseits sind die Zapfen, also die farbsensitiven Rezeptoren auf der Netzhaut, in drei unterschiedlichen Bereichen sensitiv, die grob den Farbtönen «Blau» (kurzwellig), «Grün» (mittel) und «Rot» (langwellig) entsprechen. ${ }^{25}$ Dieser Befund wird mit der sogenannten Dreifarbentheorie nach Thomas Young und Hermann von Helmholtz beschrieben. Daraus schloss übrigens James Clerk Maxwell (I86I), dass es möglich sein muss, «diese Aufspaltung photographisch durch drei sukzessive Aufnahmen mit den Grundfarbenfiltern Blau, Grün und Rot nachzuahmen», ${ }^{\mathbf{2 6}}$ und legte damit den Grundstein der meisten bis heute üblichen Farbverfahren in Film und Fotografie sowie auch im analogen und digitalen Video, die aus einer additiven oder subtraktiven Mischung von drei Primärfarben bestehen.

In Ergänzung dazu ist die Gegenfarbtheorie nach Ewald Hering (1874) ${ }^{27}$ zu berücksichtigen, die eine Verrechnung der Potenziale aus den Zapfen annimmt, an denen exzitatorische und inhibitorische Prozesse beteiligt sind. Dieser Verrechnungsprozess begründet die intuitive Empfindung, dass bestimmte 
Farbpaare - die Komplementärfarben - als Gegensätze wahrgenommen werden, nämlich «rot - grün», «blau - gelb» und «schwarz - weiß». ${ }^{28}$ Die Gegenfarbtheorie erklärt außerdem, warum Gelb neben Rot, Grün und Blau als Primärfarbe gilt. Die Zonentheorie des Physiologen Johannes A. Kries integriert die Dreifarben- und die Gegenfarbtheorie, indem sie die beiden Ansätze als komplementär beschreibt, wobei die Dreifarbentheorie den zentralen Prozess in der Retina, die Gegenfarbtheorie die neuronale Verarbeitung erfasst. ${ }^{29}$ So ist eine Einteilung des sichtbaren Spektrums der elektromagnetischen Wellen in Grundzügen bereits durch fundamentale physiologische Eigenschaften des visuellen Systems angelegt.

In einer bahnbrechenden Studie hat Eleanor Rosch ${ }^{30} \mathrm{zu}$ Beginn der I970er Jahre durch einen interkulturellen Vergleich zwischen der westlichen, in diesem Fall amerikanischen Kultur, und einer Kultur, die über lediglich zwei Farbbegriffe für Hell und Dunkel verfügte, die Dani in Neu Guinea, einen Beleg dafür geliefert, dass das Wahrnehmungssystem selbst, unabhängig von der sprachlichen Differenzierung und der Akkulturation, für grundlegende Strategien der Kategorisierung zuständig ist. Rosch nennt sie deshalb «natürliche Kategorien» (natural categories), eben weil sie kulturunabhängig funktionieren und demnach auf physiologische Gegebenheiten verweisen. Sie sind somit nicht arbiträr, sondern universell. In einer Studie zur Universalität von Farbbegriffen stellten Brent Berlin und Paul Kay ${ }^{31}$ I 969 fest, dass in verschiedenen Kulturen ähnliche Farbtöne als typisch für einen Farbbegriff wahrgenommen werden. Sie nannten diese prototypischen Farben focal points. Weiter zeigten ihre Untersuchungen, dass weltweit I I Farbbegriffe (basic color terms) vorkommen, die drei achromatische (Weiß, Grau, Schwarz) und acht chromatische Töne umfassen, und sie postulierten, dass die Unterscheidungen evolutionär in einer festen Ordnung auftreten. ${ }^{32}$ Wie Rosch jedoch zeigen konnte, besitzen die vier Grundfarben einen deutlich signifikanteren Prototypenstatus als die Mischfarben. Dieser Befund deckt sich gleichermaßen mit unserer Intuition wie mit den eingangs aufgeführten psychophysischen Grundlagen des Farbensehens.

So gibt es also sehr stabile zentrale Farbtöne und weniger stabile in den Übergangszonen dazwischen, wo mithin auch die Grenzen unscharf werden. Dazu sind mehrere Anmerkungen zu machen. Zunächst macht diese Einsicht deutlich, dass unsere Wahrnehmung dazu tendiert, kontinuierliche Phänomene aufzuspalten in Zonen von verdichteter Signifikanz sowie in Randzonen, in denen die Grenzziehungen zunehmend arbiträr werden.

Aus epistemologischer Perspektive noch bedeutsamer sind die Gründe für diese Tendenz. Nelson Goodman, der sich überaus scharf mit dem Zusammenhang zwischen Wahrnehmen und Erkennen beschäftigt hat, sieht in diesem Ordnungstrieb im Wesentlichen ein ökonomisches Prinzip des perzeptiven und kognitiven Umgangs mit großen Materialmengen. ${ }^{33}$ Gemäß Goodman ist eine Wechselwirkung zwischen Wahrnehmung und Identifikation am Werk, in denen beide Vorgänge einander bedingen. Tatsächlich muss man davon aus-
28 Kebeck, Bild und Betrachter, $45 \mathrm{f}$.

29 Welsch et al., Farben, 227.

30 Eleanor Rosch, Natural Categories, in: Cognitive Psychology, Nr. 4, $1973,328-350$.

31 Brent Berlin, Paul Kay, Basic Color Terms. Their Universality and Evolution, Berkeley (University of California Press) 1969.

32 1) Schwarz, Weiß 2) Rot 3) Gelb, Grün 4) Grün, Gelb 5) Blau 6) Braun 7) Rosa, Violett, Orange, Grau.

33 Nelson Goodman, Languages of Art, Indianapolis (Hackett Publishing Company) 1976, und Nelson Goodman, Ways of Worldmaking, Indianapolis (Hackett Publishing Company) 1990 [1978], $20 f f$. 
34 Zollinger, Farbe, 137. 35 Eleanor Rosch, Principles of Categorization, in: Eleanor Rosch, Barbara B. Lloyd (Hg.), Cognition and Categorization, Hillsdale NJ (Lawrence Erlbaum) 1978, 27-48.

36 Hahn, Filmprotokoll Revisited, 129. gehen, dass efferente und afferente Reiz- und Informationsverarbeitung ständig ineinandergreifen. Daraus resultieren zwei Grundprinzipien der ordnenden Tätigkeit im Umgang mit kontinuierlichen Stimuli, nämlich einerseits eine generalisierende und eine dichotomisierende Form. So werden ähnliche Wahrnehmungsinhalte unter einem gemeinsamen Übergriff als ähnlicher wahrgenommen, als sie tatsächlich sind - in unserem Fall zwei unterschiedliche Rottöne -, verschiedene Reize hingegen als deutlicher getrennt. Es findet ein Clustering statt, indem «verwandte Phänomene zu Kategorien zusammengefasst werden können». ${ }^{34}$ Dies wird dort evident, wo die Grenzen der Kategorienbildung unscharf werden und deshalb eine Entscheidung fordern, so im Übergang von Orange zu Rot. Jedes ordnende System ist deshalb durch Tilgung und Ergänzung gekennzeichnet.

Mit diesem elementaren Bedürfnis des Menschen, Kategorien zu bilden, befasst sich Eleanor Rosch in einer späteren Studie. ${ }^{35}$ Neben dem ökonomischen Prinzip der Komplexitätsreduktion, das für sie ein entscheidendes Movens jeder Kategorisierung bildet, weil eine Übereinstimmung der wahrgenommenen Struktur und den daraus abgeleiteten Kategorien den geringsten kognitiven Aufwand nach sich zieht, stellt sie dennoch eine Tendenz zu möglichst genauer Unterscheidung fest. Diese Tendenz bringt neben der horizontalen eine hierarchische Differenzierung mit sich, indem die groben, abstrakteren Einheiten der übergeordneten Stufen auf untergeordneten Stufen feiner aufgelöst werden. Es handelt sich dabei also um ein verzweigtes Netzwerk mit einer hierarchisch organisierten Baumstruktur, das bezogen auf die Farben auf der obersten Stufe die achromatischen von den chromatischen Farben unterscheiden würde, anschließend die Primärfarben und diese wiederum aufgeteilt in weitere Abstufungen wie Bordeauxrot, Karmesinrot, Scharlachrot, Rotorange usw.

Epistemologisch bedeutsam ist an diesen Befunden, dass sie einen engen Austausch physiologischer und kognitiver Operationen annehmen, die aber offenbar weitgehend kulturunabhängig zu funktionieren scheinen. Zur Operationalisierung dieser Kategorien für die computergestützte Analyse wären demnach Methodiken notwendig, die sich an der ground truth ${ }^{36}$ orientieren, mithin einen Austausch zwischen natürlichsprachlicher Beschreibung von Filmfarben und ihrer im Weiteren zu beschreibenden standardisierten Erfassung zu gewährleisten.

Über die natürliche Kategorienbildung hinaus - das dürfte aus der vorhergehenden Darstellung klar geworden sein - ist es notwendig, Systeme zu entwickeln und Standardisierungen vorzunehmen, die zwar die Fundamente der intuitiven Segmentierung aufgreifen, aber detaillierter ausdifferenzieren und mit festen, messtechnisch zu bestimmenden Werten verbinden.

Denn es geht ja in der wissenschaftlichen Analyse von materiellen Farbinformationen des Films oder auch (audio-)visueller Medien darum, genaue Werte zu ermitteln, aber in Hinsicht auf die ästhetische Dimension dieser Werte. 
Diese Dimension wiederum ist gegeben durch die Eigenheiten des Wahrnehmungssystems.

Seit Jahrtausenden haben sich in Kunst und Wissenschaft Farbsysteme entwickelt, die sich einer solchen Standardisierung widmen. ${ }^{37}$ Aufschlussreich an der Entwicklung der Farbsysteme, die immer auch in entsprechende Visualisierungen mündeten, ist die Evolution von linearen zu flächigen und am Ende zu räumlichen Modellen. Sie alle sind mit Sybille Krämer als «operative Bilder» ${ }^{38}$ zu verstehen, die als Vermittlungsinstrumente der Erkenntnis dienen und deren epistemologischen Status ich im Abschnitt «Auswertung» detaillierter diskutieren werde.

Nun sind Systeme, welche eine Segmentierung und eine Standardisierung aller dieser Parameter zur Erfassung und Beschreibung von Farbe vornehmen, zwar eine Grundlage für die Vermessung von Farbinformationen - und darüber hinaus zur Darstellung von Harmonie- und Dissonanzbeziehungen, die später eine Rolle spielen werden - aber sie berücksichtigen die wahrnehmungsseitigen Grundlagen kaum oder jedenfalls nicht ausreichend.

So dient das I93 I von der Commission Internationale de l'Eclairage entwickelte CIE-Normvalenzsystem nach wie vor in verschiedenen kolorimetrischen Anwendungen als Referenzsystem. Es hat jedoch den entscheidenden Nachteil, dass die Verteilung der Farbwerte nur ungenau mit der Wahrnehmung korreliert. ${ }^{39}$ Deshalb sind Systeme gefordert, die das Prinzip der visuellen Gleichabständigkeit erfüllen, also perzeptuell so gleichmäßig sind, dass Differenzen in der Darstellung auch Unterschieden in der Wahrnehmung entsprechen.

Dafür wäre das Munsell-Farbnotations-System ${ }^{40}$ denkbar, ein hinreichend gleichabständiges, bereits segmentiertes System, das der Maler Albert Henry Munsell ${ }^{41} \mathrm{zu}$ Beginn des 20. Jahrhunderts entworfen hat und zwar allein basierend auf seinen eigenen Empfindungen. Es ordnet die Parameter Farbton (bue), Buntheit (chroma) und Wert (value, für die Helligkeit) und wurde sowohl von Rosch als auch von Berlin und Kay zur Standardisierung ihrer Versuche angewandt.

Von Munsells Ansatz ausgehend hat die CIE 1976 das CIE L*a*b* Farbsystem ${ }^{42}$ durch Transformation aus den XYZ-System als Standard empfohlen. Dieses System erfüllt annähernd sämtliche Anforderungen an ein robustes Modell, das sowohl objektiv messbare Informationen geräteunabhängig erfasst, als auch den Gegebenheiten der Wahrnehmung entspricht.

\section{Erfassen von Proportionen}

Insgesamt ist die Farbfrage aber komplexer als die bisher besprochenen Aspekte vermuten lassen, und zwar aus systematischen Gründen. Denn Wahrnehmungsinhalte werden zwar vom visuellen System punktuell erfasst - durch die einzelnen Sinneszellen -, aber diese einzelnen Daten werden, wie die Gegenfarbentheorie aufzeigt, unmittelbar in einen Zusammenhang gestellt. Sie beeinflussen einander also $\mathrm{ab}$ einer sehr frühen Stufe der Integration gegenseitig. Daraus
37 Siehe Narciso Silvestrini, Ernst Peter Fischer, Farbsysteme in Kunst und Wissenschaft, Köln (DuMont) 1998. Eine der umfangreichsten Quellen ist die Website http://www.colorsystem. com/, welche die Publikation von Silvestrini und Fischer aufbereitet. Etwas befremdlich sind in Buch und Website die Darstellungen der Farbwerte durch Farbstiftzeichnungen auf texturiertem Hintergrund (der auf der Website zusätzlich gelblich erscheint), die mit ihrem halbtransparenten Auftrag den Farbeindruck maßgeblich beeinflussen; zusätzlich sind die Farbnamen in Italienisch aufgeführt. Eine weitere Quelle ist Welsch et al., Farben, $115 \mathrm{ff}$.

38 Sybille Krämer, Operative Bildlichkeit. Von der «Grammatologie» zu einer «Diagrammatologie»? Reflexionen über erkennendes Sehen, in: Martina Heßler, Dieter Mersch (Hg.), Logik des Bildlichen. Zur Kritik der ikonischen Vernunft, Bielefeld (Transcript Verlag) 2009, 94-122.

39 Siehe Kritik von Douglas L. MacAdam (1944), in: Silvestrini, Fischer, Farbsysteme in Kunst und Wissenschaft, 1998, 133 f.

40 http:|/dba.med.sc.edu/price| irf/Adobe_tg/models/munsell.html, gesehen am 5.9.2011.

41 Albert Henry Munsell, A Color Notation, Boston (G. H. Ellis Co.) 1905, http:/|books.google. com/?id=PgcCAAAAYAAJ, gesehen am 27.2.2011, und Albert Henry Munsell, The Atlas of the Munsell Color System, Boston 1915.

42 http:|/dba.med.sc.edu/price/irfl Adobe_tg/models/cielab.html, gesehen am 5.9.2011. 
folgt einer der Hauptsätze jeder Wahrnehmungstheorie, nämlich dass Wahrnehmung ganzheitlich und relativ funktioniert. An ein sinnvolles Segmentierungs- und Standardisierungssystem muss deshalb zwingend ein nächster Schritt anschließen, der die einzelnen Daten wieder in einen Zusammenhang stellt, also synthetisiert. In seiner Schrift Interaction of Color. Grundlegung einer Didaktik des Sebens ${ }^{43}$ betont der Maler und Bauhaus-Theoretiker Josef Albers anhand seiner didaktisch-experimentellen Studien, wie groß die Unterschiede zwischen der physikalischen Beschaffenheit der Farben und ihrer wahrnehmungspsychologischen Effekte sind, die aus dieser holistischen, relationalen Verarbeitung resultieren. Man nimmt weniger absolute Werte wahr, sondern die Verhältnisse der Farben untereinander, ähnlich wie in der Musik, in der nur sehr wenige Menschen mit einem absoluten Gehör diesem Netz von Beziehungen der Reize untereinander entkommen. Es sind dies insbesondere eine Reihe von nichtlinearen Effekten, die aus dem Zusammenspiel von Farben entstehen und die Farben in ihrer Wirkung untrennbar beeinflussen.

Zwar können wir Farbwerte von Filmmaterialien kolorimetrisch objektiv erfassen und in den CIE $\mathrm{L}^{*} \mathrm{a}^{*} \mathrm{~b}^{*}$-Farbraum projizieren, um eine objektive Sammlung von Daten anzulegen. Um aber die ästhetische Dimension wieder in den Vordergrund zu rücken, müssen in der Auswertung die Relationen der Farben untereinander sowie der Einfluss der Betrachtungsbedingungen berücksichtigt werden, die von der chromatischen Adaptation aufgefangen werden. Aus dieser Anpassungsfähigkeit des visuellen Reizverarbeitungssystems resultiert die sogenannte Farbkonstanz des menschlichen Wahrnehmungssystems, weshalb reife Bananen unter Licht mit unterschiedlicher Farbtemperatur immer gelb erscheinen. ${ }^{44}$ Die Empfindung von Weiß ist also immer relativ im Hinblick auf die Umgebung. Diese Eigenheit wird durch den Weißabgleich in digitalen und elektronischen Aufnahmesystemen nachempfunden, der die Farbtemperatur des Lichts neutralisiert, indem ein Weißpunkt im Aufnahmesystem als Referenzelement für Weiß definiert wird. Im fotochemischen Prozess muss die Farbtemperatur stets berücksichtigt werden, entweder in der Wahl des entsprechenden Materials für Tages- oder Kunstlicht oder durch entsprechende Filter.

In seiner als Retinex-Theorie ${ }^{45}$ bezeichneten Hypothese postulierte Edwin H. Land, ${ }^{46}$ der Erfinder der Polaroidkamera, dass das visuelle System im Zusammenspiel zwischen der basalen Reizverarbeitung durch die Zapfen in der Netzhaut mit höheren kognitiven Prozessen die Farbverschiebung durch die

43 Josef Albers, Interaction of Color. Grundlegung einer Didaktik des Sehens, Köln (DuMont Schauberg) 1970 [1963].

44 Kebeck, Bild und Betrachter, 2oof., und Zollinger, Farbe, 113 f.

45 Aus Retina und Cortex zusammengesetztes Kofferwort.

46 Edwin H. Land, The Retinex Theory of Color Vision, in: Scientific American, Bd. 237, Nr. 6, 108-128. Farbtemperatur des Lichts herausrechnet, indem es die Informationen aus dem Rot-, Grün- und Blau-Kanal einer statistischen Analyse unterwirft. Von besonderer Bedeutung sind dabei die Gradienten, die Übergänge zwischen Farbflächen. Mit ihren Experimenten, die sie anhand von «Mondrian» genannten Farbtafeln durchführten, konnten Land und seine Mitarbeiter zeigen, dass die Farbkonstanz unabhängig vom Abbildungsinhalt funktioniert.

Wahrnehmungspsychologisch ebenso bedeutsam ist der Simultankontrast, der verdeutlicht, dass Farbtöne in Abhängigkeit von der sie umgebenden Farb- 
fläche wahrgenommen werden; so erscheint ein mittleres Grau in einer weißen Umgebung dunkler als in einer schwarzen Umgebung. Noch ausgeprägter ist dieser Effekt, wenn sich die beteiligten Farben komplementär - oder nach Johannes Itten ${ }^{47}$ leicht verschoben dazu - zueinander verhalten wie ein sattes, rotes Quadrat inmitten eines lindgrünen Feldes. Treten diese Kombinationen in zeitlicher Sequenz auf, ergibt sich ein ähnlicher Effekt, der sich Sukzessivkontrast nennt.

$\mathrm{Zu}$ erfassen wären also auch die Beziehungen der Farben untereinander, also das Zusammenspiel der Farben oder - wie es Christine N. Brinckmann genannt hat - die Farbakkorde. ${ }^{48}$ Im Unterschied $\mathrm{zu}$ den objektivierbaren Parametern ist dieses Feld hochgradig durch kulturelle Faktoren bestimmt. Bei den historisch bedeutsamen Versuchen, diese Beziehungen systematisch zu erfassen, waren normative Zielsetzungen am Werk. Es ging - anders ausgedrückt - darum, optimale Harmonien zu entwerfen und deren Qualität empirisch zu begründen. So war Johann Wolfgang von Goethes Farbenlebre ${ }^{49}$ einer deutlich metaphysischen Dimension von sinnlich-sittlicher Bedeutung verpflichtet und - durchaus mit Parallelen zu Konzeptionen der Antike unter anderem bei Aristoteles - zwischen den Polen Helligkeit (Gelb) und Dunkelheit (Blau) angelegt. Er entwarf ein System der Harmonie, das weniger die physikalischen oder physiologischen Grundlagen der Farben berücksichtigte, als vielmehr eine Farbsymbolik anhand von psychologisch motivierten Zugängen zu den mit den Farbtönen assoziierten Empfindungen begründete. Damit stellte er sich in seinen skizzenhaften Reflexionen zur Farbe explizit gegen die naturwissenschaftlich fundierte Farbtheorie Newtons, die sich - wie Gage anmerkt - «bis zum I9. Jahrhundert auf die Auseinandersetzung mit der Farbe in der Kunst auswirkte».50 Jonathan Crary schreibt deshalb Goethes unsystematischen Betrachtungen zur Farbe das Verdienst zu, den eminent subjektiven Charakter der Farbwahrnehmung in den Fokus zu rücken und den Betrachter als aktiven, autonomen Produzenten seiner eigenen visuellen Erfahrung zu beschreiben. ${ }^{51}$ Mit dieser Haltung - so Crary weiter - bereite er den Boden für eine modernistische Konzeption des Betrachters, die er mit Michel Foucault ${ }^{52}$ an der Schwelle zum i9. Jahrhundert festsetzt. Radikaler und wesentlich systematischer als Goethe schrieb Arthur Schopenhauer in seiner Abhandlung Über das Sebn und die Farbe (1816) ${ }^{53}$ die subjektiven Farbempfindungen erstaunlich detailliert dem physiologischen Wahrnehmungsapparat zu. Bezugnehmend auf Niklas Luhmann ${ }^{54}$ könnte man diesen Wandel auch als einen Wandel hin zu einem Beobachten zweiter Ordnung einstufen. Es geht damit nicht nur um das Sehen als Grundlage der Wissensproduktion, sondern auch um die Beschäftigung mit den Bedingungen des Beobachtens selbst. Und es sind - wie erwähnt - genau diese Bedingungen, die von Psychophysikern in der 2. Hälfte des I9. Jahrhunderts systematisch experimentell untersucht wurden, um die subjektive Wahrnehmungsleistung mit objektiven Kriterien zu operationalisieren.
47 Johannes Itten, Kunst der Farbe, Ravensburg (Ravensburger Buchverlag) 1970 [1961], $49 \mathrm{f}$.

48 Christine N. Brinckmann, Dramaturgische Farbakkorde, in: Thomas Koebner, Thomas Meder (Hg.), Bildtheorie und Film, München (edition text + kritik) 2006, 358-380.

49 Johann Wolfgang von Goethe, Zur Farbenlehre, Weimar 1810, http:II www.textlog.delgoethe_farben.html, gesehen am 27.2.2011.

50 John Gage, Kulturgeschichte der Farbe. Von der Antike bis zur Gegenwart, Ravensburg (Ravensburger Buchverlag) 1993, 191.

51 Jonathan Crary, Techniques of the Observer. On Vision and Modernity in the 19th Century, Cambrige MA (MIT Press) 1990,69 .

52 Michel Foucault, Die Ordnung der Dinge, Frankfurt M. (Suhrkamp) 1971 [frz. Les Mots et les choses, 1966].

53 Arthur Schopenhauer, Über das Sehn und die Farben. Eine Abhandlung, Leipzig (F.A. Brockhaus) 1870 [1816]. 54 Niklas Luhmann, Die Realität der Massenmedien, Wiesbaden (VS) 1995. 
55 Itten, Kunst der Farbe.

56 Insbesondere die DVDEditionen zeigen einen wenig standardisierten Umgang mit der Farbkodierung, sodass DVDs als Quellen für die Farbbetrachtung sehr problematisch, wenn auch in den meisten Fällen unumgänglich sind, da es eher selten möglich ist, auf Filmkopien zurückzugreifen. Noch deutlicher als in den DVDEditionen dürfte die Variabilität auf den zahlreichen Internetplattformen ausfallen, wobei dort - unter anderem aufYouTube - derzeit ein Quantensprung zu höheren Auflösungen zu beobachten ist, welche die Standardauflösung von DVDs hinter sich lassen.

57 Peter Geimer, Was ist kein Bild? Zur 'Störung der Verweisung, in: Peter Geimer (Hg.), Ordnungen der Sichtbarkeit. Fotografie in Wissenschaft, Kunst und Technologie, Frankfurt M. (Suhrkamp) 2002, 313-340, hier 319.

58 Erik Reinhard, Greg Ward, Sumanta Pattanaik, Paul Debevec, High Dynamic Range Imaging. Acquisition, Display, and Image-based Lighting, Amsterdam (Elsevier/Morgan Kaufmann) 2006 .
Farbrelationen sind also in einem komplexen Feld zwischen wahrnehmungsbedingten Nichtlinearitäten und kulturell determinierten, einem historischen Wandel unterworfenen Konzeptionen von ästhetischer Produktion anzusiedeln.

In einer projektierten Vermessung der Farbrelationen ginge es jedoch nicht darum, Normen zu entwickeln, sondern bereits bestehende individuelle sowie historische oder materialgebundene Anordnungen systematisch zu erfassen und zu reflektieren. Eine gute, wenn auch nicht erschöpfende Grundlage dafür bilden die Kontraste, die der Bauhauskünstler und Didaktiker Johannes Itten ${ }^{55}$ auf der Grundlage von Vorarbeiten seines Meisters an der Stuttgarter Kunstakademie, Adolf Hölzel, entwickelt hat.

Ittens Absicht war es ebenfalls, ein normatives System zu entwerfen, dessen Begründung durch einen deutlich esoterischen Einschlag geprägt ist, der sich aus seiner alternativen Lebensweise erklärt. Das Spannungsfeld zwischen objektiven und metaphysischen oder symbolischen Dimensionen von Farbe zieht sich somit wie ein roter Faden bis in die jüngste Vergangenheit durch die systematische Beschäftigung mit diesem Gegenstand. Aber im Unterschied zu anderen normativen Ansätzen eignet sich Ittens Ansatz auch für die Analyse und ist darin erstaunlich nüchtern, weil die Parameter entsprechend der kolorimetrisch bestimmbaren Dimensionen Farbton, Buntheit, Helligkeit skalierbar sind. Würden die analysierten Farbvalenzen also in ein wahrnehmungsgerechtes Farbsystem projiziert, ließen sich Kontraste unmittelbar daraus ablesen und systematisieren.

Kritisch anzumerken bleibt, dass ein solches System sehr schablonenhaft bleiben müsste und gerade wahrnehmungsbedingte Nichtlinearitäten schwer zu berücksichtigen wären. Zur medialen Rezeptionssituation im Kino ist zudem festzustellen, dass sie nur in Maßen normiert ist. Hingegen ist die Variabilität der medialen Dispositive sehr groß und hat einschneidende Konsequenzen für den Farbeindruck. ${ }^{56}$ Dem materialbasierten Aspekt, welcher der systematischen Analyse eher zugänglich ist, steht somit eine performative Dimension gegenüber, die durch eine Vielzahl von Faktoren bestimmt ist; neben technologischen Eigenheiten der Dispositive selbst sind es kulturell befrachtete Einflüsse der sozialen Rezeptionssituation. Es wird - wie Peter Geimer es ausdrückt - «die Materialität des [...] Bildes in ein Netzwerk sozialer Praktiken eingeflochten.» ${ }^{57}$

Weiter hat die Diskussion zu den natürlichen Kategorien nach Rosch gezeigt, dass es eine Tendenz zur Mitte hin gibt, die man möglicherweise gerade dann berücksichtigen müsste, wenn man versucht, die Relationen der Farben untereinander wieder einzubeziehen. So scheinen die Grundfarben eine andere Dominanz aufzuweisen als Mischfarben. Außerdem sind es nicht nur die vorkommenden Farben selbst, die ausschlaggebend sind, sondern deren quantitative sowie örtliche Verteilung im Bild. Während Filter zur Flächenbestimmung von Bildinformationen schon andernorts im Einsatz sind, zum Beispiel in der Berechnung von Lichtwerten aus Fotografien, ${ }^{58}$ dürften bildkompositorische 
Aspekte schwieriger zu standardisieren und in ein computergestütztes Analysetool zu implementieren sein.

Zusätzlich müsste ein zeitlicher Filter eine Grundlage für die Abtastungsfrequenz liefern, also festlegen, wie häufig Stichproben genommen werden, idealerweise in Abhängigkeit von Szenenwechseln. Die Filterung, das heißt die Segmentierung des Materials in seine Komponenten, muss also neben dem eigentlichen Analysegegenstand, nämlich der Farbdistribution, auch die dem Film und allen audio-visuellen Medien eigene räumlich-zeitliche Organisation berücksichtigen, eine Anforderung, mit deren grundlegender Problematik wir uns am Ende nochmals beschäftigen werden.

Als Resultat der Analyse von Farbakkorden wären zwei Darstellungsformen denkbar: Eine Farbpalette, welche die wichtigsten Farben visuell darstellt, ${ }^{59}$ und eine dreidimensionale Darstellung der Kontraste in einem der (annähernd) wahrnehmungsgerechten Farbsysteme, also im CIE L*a*b* oder im Munsell Color System.

\section{Auswertung}

Medientechnisch und filmhistorisch gesehen ist Farbe im Film (und in den bildlichen Medien) eine nachgeschaltete Qualität, die sich erst ab den I950er und ig6oer Jahren als Standard etablierte. Medientheoretisch bedeutsam sind die grundlegenden Fragestellungen, die sich der Verbreitung von Farbprozessen entgegenstellten. Denn es erwies sich als überaus schwierig, technische Verfahren zu entwickeln, welche das kontinuierliche Spektrum der sichtbaren elektromagnetischen Wellen abbildeten. Sie führen uns zurück in sämtliche bereits dargestellten Problembereiche der Segmentierung und Standardisierung, die sich in der technischen Domäne gleichermaßen stellen wie in der Wahrnehmungs- und Kulturtheorie der Farbe.

In seiner kurzen, aber hervorragenden Geschichte der gemeinsamen Entwicklung messtechnischer Verfahren in der psychophysischen Domäne und chemisch-physikalischer Techniken der Farbwiedergabe in Fotografie und Film stellt der Wahrnehmungspsychologe Ruggero Pierantoni ${ }^{60}$ die unglaublichen Hürden plastisch dar, welche aus dem notwendigen Zusammenspiel aller Komponenten für die Farbdarstellung resultierten. Diese Probleme sollen uns nicht im Detail interessieren. Viel wichtiger sind ein paar daraus resultierende grundlegende Einsichten. Neben der Einsicht, dass Techniken der Farbwiedergabe ohne das entsprechende Wissen über die physiologischen Grundlagen des Farbensehens undenkbar gewesen wären, wird vor allem deutlich, dass es ungeheuer schwierig war, Prozesse zu entwickeln, welche Farben in ihrer ursprünglichen Vielfalt und komplexen Zusammensetzung wiederzugeben in der Lage sind. Daraus resultiert, dass mimetische Prozesse der Farbaufnahme historisch gesehen nach den autonomen Verfahren auftraten. Oder mit anderen Worten: Die frühen Verfahren wie Virage und Tonung, Schablonen- und
59 Ein System zur Extraktion von fünfteiligen Farbpaletten aus Bildern wird von Adobe im Internet angeboten, http:|/kuler.adobe.com/\#create| fromanimage. Es basiert auf der manuellen, punktuellen Analyse von Farbwerten, wobei das System eine automatische Analyse vorschlägt.

60 Ruggero Pierantoni, One Colour for All Seasons. Knowledge, Measures and Myths at the Beginning of Cinem, in: Luciano Berriatúa et al. (Hg.), Tutti i colori del mondo. II colore nei mass media tra 1900 e 1930, Reggio Emilia (Edizioni Diabasis) 1998, 135-140. 
61 Gage, Kulturgeschichte der Farbe, 205 f.

62 Es ist unerlässlich, an dieser Stelle darauf hinzuweisen, dass die digitale Bildaufzeichnung gleichermaßen durch einen physikalischen Prozess materieller Lichtaufnahme begründet ist wie die analogen Prozesse, denn die Sensoren - CCD oder CMOS - reagieren auf das einfallende Licht zunächst analog. Erst in einem daran anschließenden Prozess werden diese Informationen binär kodiert und damit in die digitale Domäne gewandelt.
Handkoloration sind gezwungenermaßen Techniken, welche ein symbolisches, weil autonomes und komplett arbiträres Verhältnis von Farbe und Gegenstand mit sich brachten, das nur auf der freien Interpretation der für den Färbungsvorgang zuständigen Instanzen beruhen konnte, nicht aber auf jenem automatischen oder halbautomatischen Vorgang der direkten Aufzeichnung von Licht, die André Bazin als für den Film konstitutiv beschrieb. Kulturhistorisch ist dieser Umstand bedeutsam, denn in der Malerei ist - wie John Gage in seiner Kulturgeschichte der Farbe eindringlich beschreibt - in der zweiten Hälfte des I 9. Jahrhunderts die zunehmende Autonomie der Farbgestaltung ebenfalls im Kontext der psychophysischen Erforschung des visuellen Systems zu verstehen. ${ }^{61}$ Das Bestreben dieser Bewegungen in der bildenden Kunst seit dem Impressionismus war es, einen Zugang zu dieser unmittelbaren Wahrnehmungsqualität von Farbe zu erlangen, sozusagen ein unschuldiges Auge, das die Bürden der Tradition und die Anforderungen des Realismus gleichermaßen zu sprengen in der Lage wäre. Noch deutlicher manifestiert sich diese Entwicklung in der später einsetzenden abstrakten Malerei, wo die Wahrnehmungsdimension als grundlegendes Prinzip in den Vordergrund rückte und nach allgemeinen Prinzipien der Komposition verlangte, die - wie eingangs dieses Textes mit Kandinsky erwähnt - wiederum eine verstärkte Bewegung hin zu Vermessung und Systematisierung der ästhetischen Parameter auslöste.

Mediengeschichtlich sowie ästhetisch bedeutsam ist die Einsicht, dass praktisch alle Farbverfahren - photochemische, elektronische und digitale - auf einer Zerlegung des kontinuierlichen Farbspektrums auf meist drei Frequenzbänder beruhen. In diesen Techniken sind also implizite, empirisch fundierte Prinzipien schon wirksam. Sie privilegieren grundsätzlich die mimetische gegenüber der autonomen Farbbehandlung, weil sie einen wenn auch fragmentierten, so doch durch analoge ${ }^{\mathbf{6 2}}$ Verknüpfungen bestimmten Prozess zwischen Urbild und Abbild implementieren, der auf objektiven Kriterien zur Bestimmung der relevanten Parameter beruht. Ein analytisches Verfahren, das sich dieser Prinzipien bedient, würde aus dieser Sicht an die angewandten Techniken anschließen.

Wenn ein webbasiertes, weltweit zugängliches Framework zur Verfügung stehen würde, könnte damit ein Instrument entstehen, die filmhistorisch bedeutsamen Bewegungen in ihren Eigenheiten zu erfassen. Es würde damit ein Open-Source-Instrument geschaffen, das die dynamische Interaktion verschiedener an der Analyse beteiligter Akteure erlaubt und wiederum im Rahmen eines solchen kollaborativen Zugriffs laufend in seiner Tauglichkeit überprüft und angepasst werden könnte. Computergestützte Analyse erfordert jedoch unbedingt, dass die hier geschilderten Fragestellungen der für ein solch global zugängliches Unterfangen notwendigen Standardisierung hinreichend wahrnehmungstheoretisch wie kulturgeschichtlich reflektiert worden sind.

Damit soll allerdings nicht postuliert werden, dass ein solches Analyseinstrument an sich erschöpfende Ergebnisse liefern und damit eine Art Weltformel für die Erfassung jedes individuellen Werks zur Verfügung stellen könnte. 
Vielmehr müsste man an die semi-automatische Datenerhebung für jede Einzelanalyse einen umfassenden Kontextualisierungsschritt anschließen, der auf Vorarbeiten in Kunstgeschichte und Filmwissenschaft anknüpft und die Ergebnisse sowohl historisch wie auch ästhetisch situiert.

Schließlich ist Farbe in unserer Erfahrung und in der Bildwahrnehmung traditionell mit der Objektwahrnehmung verbunden und diese Dimension wird von automatischen Erfassungssystemen grundsätzlich ausgeklammert. Dazu gehören auch charakteristische Eigenheiten von abgebildeten Materialien wie Texturen, Muster und Reflexionsverhalten, welche die Ästhetik der Farbwirkung entscheidend beeinflussen. Insbesondere die optische Verarbeitung der profilmischen Welt und ihrer Objekte war immer sehr stark bestimmt durch einen differenzierten Umgang mit deren materieller Zusammensetzung, die im Zeichen der Photogénie möglichst optimal im Hinblick auf die materiellen Eigenschaften des Films selbst ausgewählt worden sind. So hat beispielsweise Natalie Kalmus, die während Jahrzehnten als verantwortliche Color Consultant für Technicolor tätig war, für diesen Farbprozess ein umfangreiches normatives System optimaler Farbpaletten entwickelt, an das sich Filmproduktionen samt Kameradepartment, Ausstattung und Kostüme halten sollten. ${ }^{63}$

Auch die Unterscheidung zwischen Körper- und Lichtfarben, zwischen undurchsichtigen und transparenten Farben, wie sie besonders von Runge in seiner Farbtheorie schon etabliert worden ist, ${ }^{64}$ wird durch ein solches Analyseverfahren nicht erfasst. Was zweifellos eine Reduktion des phänomenalen Detailreichtums und einer großen damit einhergehenden Vielfalt visueller Erscheinungen des Films bedeutet, ist gleichzeitig aus epistemologischer Sicht auch ein Gewinn. Denn weil sich die Objekthaftigkeit der Welt immer in den Vordergrund der Wahrnehmung schiebt, weil unsere Erfahrung immer durch den Umgang mit der gegenständlichen Welt geprägt ist, bedeutet eine Reduktion eine Befreiung von diesen Konventionen - ähnlich der «eidetischen Reduktion» im Sinne Edmund Husserls $-{ }^{65}$ welche die Stofflichkeit des Films selbst in den Fokus nimmt und deren Wesenheit untersucht oder - anders ausgedrückt - die Darstellung nicht als Repräsentation begreift, sondern in ihrer materiellen Beschaffenheit untersucht. Damit ist diese Analyse vergleichbar mit jenem bereits geschilderten Bestreben nach einem unschuldigen Auge, das die Maler des ausgehenden I9. Jahrhunderts antrieb, in ihrer Kunst an den Bedingungen der Wahrnehmungen selbst zu arbeiten.

Jüngst hat David N. Rodowick ${ }^{66}$ mit Verweis auf den kurzen Essay «The Unattainable Text» (1975) von Raymond Bellour ${ }^{67}$ eine grundlegende Schwierigkeit aller Filmanalyse bearbeitet, dass nämlich jede Verschriftlichung des audiovisuellen Bewegtbildes notwendigerweise hinter ihrem Analyseobjekt zurückbleibt. «The solid ontological anchoring of a worked substance is grasped only with difficulty, yielding an art that, so far, leans, more than any other, on an experience of the Imaginary.» ${ }^{68}$ Einen Ausweg aus diesem Dilemma bietet die Diagrammatik als eine metaphorische Vermittlungsinstanz zwischen einer
63 Siehe Natalie Kalmus, Color Consciousness, Erstabdruck in: Journal of the Society of Motion Picture Engineers, Nr. 25, August 1935, in: Angela Dalle Vacche, Brian Price (Hg.), Color. The Film Reader, New York (Routledge) 2006.

64 Siehe Martin Roman Deppner, Zur Farbdramaturgie Douglas Sirks. Kunstrezeption im Spielfilm zwischen Farbautonomie und Farbbedeutung, in: Anne Hoormann, Karl Schawelka (Hg.), Who's afraid of. Zum Stand der Farbforschung, Weimar (Universitätsverlag), 302-334, hier 308f., und Gage, Kulturgeschichte der Farbe, 203.

65 Edmund Husserl, Phänomenologische Psychologie, Hamburg (Felix Meiner Verlag) 2003 [1925], $46 f$.

66 David N. Rodowick, The Virtual Life of Film, Cambridge MA, London (Harvard University Press) 2007.

67 Raymond Bellour, The Unattainable Text, in: Screen, Nr. 16.3, Herbst 1975, 19-27.

68 Rodowick, The Virtual Life of Film, 2007, 21. 
69 Sybille Krämer, Das Auge des Denkens. Visuelle Epistemologie am Beispiel der Diagrammatik, Zwölfte Vorlesung. Charles Sanders Peirce. Sichtbarkeit und Graphematik des Denkens, Freie Universität Berlin, WS 2009/10, ohne Seiten, Download auf http:||userpage.fu-berlin.de/ffisybkram/ pages/de/downloads.php, gesehen am 27.2.2011.

70 Christine N. Brinckmann, Filmische Farbe, als Abbild und Artefakt, in: Hans-Georg von Arburg et al. (Hg.), (Wunderliche Figuren). Über die Lesbarkeit von Chiffrenschriften, München (Fink) 2001, 187-206, hier 188 .

71 Sybille Krämer, Operative Bildlichkeit, 10of.

72 Ebd., 107.

73 Gottfried Boehm, Ikonisches Wissen. Das Bild als Modell, in: Gottfried Boehm, Wie Bilder Sinn erzeugen. Die Macht des Zeigens, Berlin (Berlin University Press) 2007, 114-140, hier 127.

74 Paul Humphreys, Extending Ourselves. Computational Science, Empiricism, and Scientific Method, Oxford (Oxford University Press) 2004, 138.

75 Raymond Bellour, Unattainable Text, 26.
Bildlogik und einer Sprachlogik. Mit der Projektion der ermittelten Daten in ein visuell zugängliches Farbsystem wird ein solcher erkenntnistheoretisch bedeutsamer Schritt von konkreter Anschaulichkeit hin zu konzeptueller Abstraktion vollzogen. Insbesondere der relationale Aspekt, der über die empirische Analyse der Farbakkorde gewonnen würde, entspricht in seiner Essenz dem Anspruch an Diagramme, «Evidenz zu stiften», indem sie nicht nur als «Visualisierungsinstrument», sondern als «Denkwerkzeug» dienen könnten, wie Sybille Krämer in ihren Ausführungen zur Diagrammatik bei Charles Sanders Peirce schreibt. ${ }^{69}$ Insofern steht die diagrammatische Auseinandersetzung mit Erkenntnissen aus der systematischen Analyse ästhetischer Strategien, die - wie erwähnt - besonders in den Avantgarden des frühen 20. Jahrhunderts schon als taugliches Instrument erachtet wurden, erkenntnistheoretisch auf einem soliden Fundament. Besonders in der filmischen Farbe wirkt, wie Christine N. Brinckmann ${ }^{70}$ schreibt, «viel Akzidentielles» mit, es «vermischen sich [...] Beiläufiges und Essentielles». Diese «Schwierigkeiten [potenzieren sich] im Film [...], wenn zur stillgestellten Komposition die Bewegung hinzukommt und wenn das Bild nicht mehr als Objekt existiert, sondern projiziert wird.» In der diagrammatischen Darstellung wird die sinnlich-ästhetische Dimension von dieser akzidentiellen, singulären Erscheinungsweise sozusagen gereinigt und in eine abstrakte, mehr der generellen Begrifflichkeit zugeordneten epistemischen Kategorie verschoben. Gerade im grafischen Element der Linie als Mittel der Visualisierung von Relationen sieht Sybille Krämer ${ }^{71}$ mit Rückgriff auf Horst Bredekamps Reflexionen zu wissenschaftlichen Darstellungen eine erkenntnistheoretisch bedeutsame Verschiebung, weil sie etwas visualisiert, «das den Sinnen gerade nicht unmittelbar, sondern auf eine andere Weise» zugänglich ist. ${ }^{72}$ Das «Bild als Modell» produziert eine «unvordenkliche Übertragung, durch die das Perzeptuelle als etwas Konzeptuelles, das Materielle als eine räumliche oder logische Bestimmung erfahren werden kann», ${ }^{73}$ es hat, anders gesagt, eine heuristische Funktion, die in der Entwicklung der Farbsysteme sehr schön hervortritt.

Mit den Mitteln der Computeranimation wäre es zudem möglich, die Raumkoordinaten der diagrammatischen Darstellung von Proportionen mit der Zeitachse zu verknüpfen und somit ein konzeptuelles Analogon zum räumlich-zeitlich organisierten Medium zu erhalten. Auch diese temporalen, dynamischen Darstellungsformen blieben notwendigerweise semantisch und formal unterdeterminiert. ${ }^{74}$ Sie lösen deshalb nicht das Problem jeder Analyse, das schon Bellour isoliert, nämlich dass sie a priori unabschließbar und damit fiktional bleibt. ${ }^{75}$ In diesem Horizont könnte die Vermessung und Transkription ästhetischer Erscheinungen einen produktiven, wenn auch keineswegs erschöpfenden Beitrag leisten. 